\title{
Alexandria - Beirut - Paris: Avantgarde und geistiger Widerstand bei Georges Schehadé und Leila Baalbaki
}

\section{Einleitung}

Dieser Artikel widmet sich der Frage, wie das französischsprachige Werk des libanesischen Autors Georges Schehadé und das arabische Euvre der libanesischen Autorin Leila Baalbaki zwischen Alexandria, Beirut und Paris zu verorten sind. Während Schehadé einen Großteil seines Werks in Paris verfasst hat, ist die Zeit Baalbakis in Paris auf die Jahre 1959 und 1960 beschränkt. Beide sind, so die These, wichtige Akteure der Avantgarde, und sie sind es, weil sie ihre Erfahrungen zwischen Ländern und Sprachen gezielt in ihren literarischen Verfahren aufzuheben suchen. Ihre poetologischen Entwürfe sollen im Folgenden als Modi geistigen Widerstands gedeutet werden: während Georges Schehadé die französische Sprache kreativ einsetzte, um Literatur aus der Logik des Nationalen herauszulösen, nutzte Leila Baalbaki die republikanische Imprägnierung des Französischen für eine ästhetische Bestandsaufnahme der traditionalistischen libanesischen Gesellschaftsordnung.

\section{Gärten ohne Land - Die Dichtung Georges Schehadés (1905-1989)}

Georges Schehadé wurde 1905 in Alexandria, Ägypten geboren. Das Land gehörte seit 1882 zum britischen Herrschaftsgebiet. Zwei Jahre vor der ersten, noch eingeschränkten ägyptischen Unabhängigkeit im Jahr 1922 zog die christlich-orthodoxe Familie zurück nach Beirut. ${ }^{1}$ Hier publizierte Georges Schehadé erste Gedichte und nahm ein Jurastudium auf. Er arbeitete zunächst für das französische Hochkommissariat im Libanon, dann für das Justizministerium und später als Generalsekretär der École Supérieure des Lettres in Beirut. Der Surrealismus sollte ein

1 Im Libanon erlebte Schehadé das Ende des Französischen Mandats (1919-1943) und ab 1945 die Unabhängigkeit.

2 Open Access. (c) 2020 Catarina von Wedemeyer, publiziert von De Gruyter. (c) BY-NC-ND Dieses Werk ist lizenziert unter der Creative Commons Attribution-NonCommercial-NoDerivatives 4.0 Lizenz. https://doi.org/10.1515/9783110679366-011 
wichtiger ästhetischer Bezugspunkt für sein eigenes Schreiben werden. 1933 lernte Schehadé in Paris so prominente Dichter wie Saint-John Perse, Max Jacob und Jules Supervielle kennen. Nachdem er 1949 nach Frankreich gezogen war, gehörte er bald zu den aktiven Mitgliedern der Gruppe um André Breton. Es war niemand anderes als Breton selbst, der Schehadés kontrovers diskutiertes Theaterstück Monsieur Bob’le 1951 in der Zeitschrift Le Figaro Littéraire verteidigte. ${ }^{2} \mathrm{Zu}$ den Bekanntschaften, die Schehadé in Paris machte, zählten Autoren des absurden Theaters, Eugène Ionesco, Samuel Beckett, aber auch Künstler wie Marc Chagall, Octavio Paz und Andrée Chédid. Auf eine Einladung Léopold Sédar Senghors reiste er 1967 nach Dakar. Es folgten Reisen nach New York und Montréal. ${ }^{3}$ In den Jahren 1969 bis 1977 lebte Schehadé wieder im Libanon, zog aber wegen des Bürgerkriegs (1975-1990) in den siebziger Jahren endgültig zurück nach Frankreich. 1986 bekam der Autor den Grand Prix de la Francophonie der Académie Française verliehen. Drei Jahre später starb Georges Schehadé in Paris. ${ }^{4}$

Insbesondere in der Zeit während des Zweiten Weltkriegs finden sich bei Schehadé immer häufiger poetische Texte, die sich dem zeitgenössischen dualistischen Denken von Heimat und Exil widersetzen. So ist in Gedicht Nummer 2 aus den Poésies II von 1948 die Rede von «Gärten ohne Land» und «Tauben ohne Nestern» ${ }^{5}$ :

Il y a des jardins qui n'ont plus de pays

Et qui sont seuls avec l'eau

Des colombes les traversent bleues et sans nids

Mais la lune est un cristal de bonheur

Et l'enfant se souvient d'un grand desordre clair

2 Vgl. André Breton in Le Figaro Littéraire über die Inszenierung von Schehadés «Monsieur Bob’le» (1951). Vgl. zu der «minor surrealist crisis», die aus dieser Parteinahme seitens Breton entstand: Francis J. Carmody: Les Poésies by Georges Schehadé. In: The French Review 26, 2 (Dezember 1952), S. 145-147, hier S. 146. <http://www.jstor.org/stable/382870> [03.08.2017]. Eine Analyse des Stücks gibt es von: Bettina Knapp: He who dreams diffuses into air.... In: Yale French Studies 29 (1962), S. 108-115.

3 Vgl. das Vorwort von Jürgen Brôcan in: Georges Schehadé: Poesie I-VII, französisch - deutsch. Übersetzung von Jürgen Brôcan. Berlin: Hans Schiler 2006, S. 5-10.

4 Vgl. zur Biografie: Heribert Becker: Surrealismus levantinisch. Lyrik zwischen Symbolismus und Surrealismus - der libanesische Dichter Georges Schehadé. 2006. <http://de.qantara.de/inhalt/ georges-schehade-surrealismus-levantinisch> [08.08.2017]. Rezension zu: Georges Schehadé: Poesie I-VII, französisch - deutsch.

5 Georges Schehadé: Poésies II (1948) - II. In: Les Poésies. Paris: Gallimard 1969, S. 58. Vgl. den Eintrag von Joel Kerdraon: Georges Schéhadé (1905-1989). In: A la lettre. <http://www.alalettre. com/schehade.php> [08.08.2017]. Vgl. für weitere Gedichtanalysen: Daniel Delas: La mémoire et l'éphémère dans la poésie de Georges Schehadé. In: Neohelicon XXXIII, 1 (2006), S. 131-137. 
Das darauffolgende Gedicht ist denjenigen gewidmet, «die fortgehen um ihr Haus zu vergessen» ${ }^{6}$. Die erste Strophe lautet wie folgt:

A ceux qui partent pour oublier leur maison

Et le mur familier aux ombres

J'annonce la plaine et les eaux rouillées

Et la grande Bible des pierres

Im selben Band findet sich ein weiteres Gedicht, das diese Deutung bestätigt. ${ }^{7}$ Auch hier werden Bilder von Exil in eine grundlegende Erfahrung von Lebenswirklichkeit transformiert, die die Menschheit jenseits des Nationalen vereint. Das Gedicht ist Charles Lucet gewidmet, der unmittelbar nach Ende des Zweiten Weltkriegs als Botschafter der provisorischen französischen Regierung in Beirut stationiert war. In den Jahren 1947-1950 vertrat Lucet die vierte Republik in Ägypten. ${ }^{8}$ Dies sind auch die Stationen im Leben Schehadés, und dies ist die Zeit, in der das folgende Gedicht des Autors entstand, datiert ist es auf das Jahr 1948.

\footnotetext{
Ils ne savent pas qu'ils ne vont plus revoir

Les vergers d'exil et les plages familières

Les étoiles qui voyagent avec des jambes de sel

Quand la nuit est triste de plusieurs beautés

Ils oublient qu'ils ne vont plus entendre

Le vent de la grille et le chien des images

L'eau qui dort sur la couleur des pierres

La nuit avec des violons de pluie

Tant de magie pour rien

Si ce n'est ce souvenir d'un autre monde

Avec des oiseaux de chair dans la prairie

Avec des montagnes comme des granges

Ô mon enfance ô ma folie.
}

Das Gedicht evoziert kleinste Fragmente maritimer Landschaft und verbindet diese mit einer allumfassenden Erfahrung des Vergessens, des Unterwegs- und Fremdseins, und im letzten Vers, mit einer zeitlichen Nostalgie. Die Erinnerung an eine «andere Welt» bleibt auch in diesem poetischen Text geographisch unbestimmt und wird als existentielle Erfahrung der Zeit gedeutet. Es ist auffällig, wie sehr sich die Dichtung Schehadés konkreten historischen Zusammenhängen

6 Georges Schehadé: Poésies II (1948), S. 59.

7 Ebda., S. 65.

8 Vgl. die Biographie von Charles Ernest Lucet im Bundesarchiv: <http://www.bundesarchiv.de/ cocoon/barch/0000/z/z1960a/kap1_12/para2_78.html>[05.08.19]. 
entzieht. Erfahrungen von Exil, Heimatlosigkeit und die Reflexion von Zugehörigkeit spielen eine zentrale Rolle in seinen Texten. Relativiert wird diese Unbestimmtheit allerdings durch die zahlreichen Widmungen an französische Schriftsteller, deren Biographien symbolisch für die politische Einstellung Schehadés stehen. Alle Widmungsträger sahen sich gezwungen aus Frankreich auszuwandern, sei es weil sie in der Résistance gekämpft oder anderweitig Widerstand gegen das Vichy-Régime geleistet hatten: Charles Ernest Lucet (1910-1990), dem das zuletzt zitierte Gedicht gewidmet ist, war 1942 vom Vichy-Regime von seinem Posten als französischer Botschafter in Washington abgesetzt worden; ab 1943 arbeitete er für die Exilregierung Charles de Gaulles in Algier und Ankara. ${ }^{9}$ Eine weitere Widmung gilt Jules Supervielle (1884-1960), der in Uruguay geboren war und eine doppelte Staatsbürgerschaft besaß. Auch seinem langjährigen Freund Saint-John Perse (1887-1975, alias Alexis Leger), widmete Schehadé Gedichte. Der Diplomat und Nobelpreisträger, der in Guadeloupe zur Welt gekommen war, wurde 1940 all seiner Ämter enthoben; im gleichen Jahr entzog man ihm die französische Staatsbürgerschaft und beschlagnahmte sein gesamtes Vermögen. ${ }^{10}$ Saint-John Perse emigrierte daraufhin in die USA, wo er 1942 den Gedichtband Exil publizierte, der in Frankreich nur unter der Hand verlegt werden konnte. ${ }^{11}$

Diese indirekten Bezugnahmen auf zeitgeschichtliche Zusammenhänge unterscheiden sich von den sehr viel eindeutigeren Positionierungen, die - auch im Sinne der Vorstellung engagierter Dichtung von Jean-Paul Sartre - zu diesem Zeitpunkt in der französischen Dichtung $\mathrm{zu}$ finden sind. Das Gleiche gilt im Übrigen für die arabischsprachige Literatur - vor allem seit der Staatsgründung Israels in eben dem Jahr, in dem dieses Gedicht entstand. Mit dem Verzicht auf konkrete historische Bezüge, so die These, widersetzt sich die Dichtung Schehadés den dichotomischen Ideologien ihrer Zeit, die sich mit dem Nahostkonflikt und während des Kalten Krieges noch verschärfen sollten.

9 Der zukünftige Präsident de Gaulle war selbst von 1929 bis 1931 in Beirut stationiert, bevor der Libanon nach 1940 durch das Vichy-Regime kontrolliert wurde.

10 Vgl. die Rede Saint-John Perses zur Annahme des Nobelpreises 1960: <http://www.nobelprize.org/nobel_prizes/literature/laureates/1960/perse-speech-fr.html> [09.08.2017].

11 Der Gedichtband «Exil» wurde 1942 in der Zeitschrift Poetry (März 1942), sowie in den Cahiers $d u$ Sud (Marseille) publiziert. In Poetry erscheint das Gedicht begleitet von einer «Note on Alexis Saint Leger», die Archibald MacLeish in Zusammenarbeit mit Saint-John Perse verfasste. Gallimard publizierte eine heimliche Ausgabe mit fünfzehn mit S.J.P. signierten Exemplaren. Vgl.: $<$ http://fondationsaintjohnperse.fr/une-vie-de-poete-et-de-diplomate/chronologie/> [10.08.2017]. 


\section{Krieg und Exil - Das absurde Theater Schehadés}

Das dramatische Werk Schehadés ist sehr viel eindeutiger $\mathrm{zu}$ fassen als das poetische Euvre des Autors. Georges Schehadé verfasste seine Texte sämtlich auf Französisch, sie wurden breit rezipiert, und einige seiner Theaterstücke wurden in den fünfziger und sechziger Jahren sogar im deutschen Sprachraum - in Zürich, München, Berlin und Bochum - gespielt. ${ }^{12}$ Ein Beispiel ist das in Zürich uraufgeführte antimilitaristische Stück Histoire de Vasco aus dem Jahr $1956 .{ }^{13}$ Das Stück kombiniert einen politischen Subtext mit poetischen Bildern und gilt als Exempel des absurden Theaters.

Bei der Geschichte des Vasco handelt es sich um ein surrealistisches Drama in sechs Akten, das während eines nicht genau spezifizierten Krieges spielt. Im Klappentext heißt es: «Cela se passe vers 1850 - en Amérique du Sud, en Allemagne ou bien en Italie, au cours d'une guerre.» ${ }^{14}$ Eröffnet wird das Stück von César, der davon lebt, sowohl seine Tochter Marguerite als auch ein paar ausgestopfte Hunde an Passanten zu vermieten. Ohne ihn zu kennen, hat Marguerite einen Traum von Vasco, dem Titelhelden des Stücks, einem Friseur, von dem sie durch den Traum weiß, dass sie ihn liebt (1. Akt). Die Szenen ihrer Suche nach dem zukünftigen Geliebten wechseln sich ab mit dessen Erlebnissen als unfreiwilliger Soldat. Vasco hatte versucht, sich zu verstecken, doch schon im 2. Akt wird er von Leutnant Septembre zwangsrekrutiert. Dieser ist dagegen, den vollkommen unerfahrenen Friseur in den sicheren Tod zu schicken, kann sich aber nicht durchsetzen: sein militärischer Vorgesetzter ist überzeugt, dass gerade ängstliche Menschen besonders strategisch handelten (3. Akt). Im 4. Akt trifft Vasco an einem Militärposten auf drei als Frauen verkleidete Männer, die erzählen, sie hätten gemütlich neben drei Soldaten der Feindesmannschaft gepicknickt, die sich wiederum als Bäume verkleidet hatten. Die Namen der Soldaten sind ebenso schwer an eine Nation zu binden wie die Verortung des Stücks insgesamt. Als Vasco den Posten erreicht, fragt Leutnant Brounst auf einmal in deutscher Sprache: «Wer da?» ${ }^{15}$ Vasco gibt sich $\mathrm{zu}$ erkennen und äußert seine Lebenseinstellung, die im bewaffneten Konflikt äußerst problematisch ist, denn sie lautet: «Je

12 Es handelt sich um die Stücke: Monsieur Bob’le (1951), La Soirée des proverbes (1954) (übersetzt als Der Sprichwörterabend), Histoire de Vasco (1956, Geschichte des Vasco) und Le voyage (1961, Die Reise), vgl. Heribert Becker: Surrealismus levantinisch.

13 Georges Schehadé: Histoire de Vasco. Pièce en six tableaux. Paris: Gallimard, 1957.

14 Ebda., Klappentext.

15 Ebda.S. 134. 
suis l'ami de tout le monde». ${ }^{16}$ Schon sein Name ist ironisch: Anders als der portugiesische Seefahrer Vasco da Gama wäre der Protagonist Vasco lieber in seinem Dorf geblieben. Erst als er mitten im Schussfeld steht, wird ihm der Ernst seiner Situation bewusst: «Pas de doute, il y a la guerre et je suis... au milieu! Il faut que je me méfie.» ${ }^{17}$ An dieser Stelle trifft Vasco auch auf Marguerite, die ihn erst als ihren Traummann und «Verlobten» erkennt als es schon zu spät ist: Inzwischen wurde der unfreiwillige Soldat von den drei vermeintlichen Kastanienbäumen (den Sergents Caquot, Paraz und Alexandre) gefangengenommen. Erst durch das Verhör versteht er, dass es sich bei dem gesuchten tapferen Kriegshelden um ihn selbst handeln muss. Da er es immer allen recht machen will und gerne neue Freundschaften schließt, betrinkt er sich mit Caquot und willigt ein, die militärischen Pläne seiner Seite zu verraten. Aufgrund mangelnder Kenntnis derselben wird er schließlich erschossen. Im 6. Akt liegt Vasco tot unter einem Baum; Marguerite, César und der Leutnant Septembre sind die einzigen, die um ihn trauern.

Das Stück stellt die Absurditäten von Heldengeschichten und von Krieg insgesamt heraus. Die Soldaten werden ins Lächerliche gezogen, die Gewalt scheint sinnlos. Histoire de Vasco löste in Frankreich eine heftige Debatte aus. Das Land hatte 1956 den blutigen Indochinakrieg gerade hinter sich und befand sich inmitten des Konflikts in Algerien (1954-1962). Antikoloniale Intellektuelle kritisierten den Umstand, dass Schehadés Drama aus politischen Gründen an das Schauspielhaus Zürich «ausgelagert» worden war, statt in Paris aufgeführt zu werden. ${ }^{18}$ Auf der anderen Seite reagierten die Anhänger eines französischen Algerien ebenso empört: Exemplarisch dafür steht folgende von Leon Treich verfasste Rezension des Stücks, sie trägt den Titel: «Histoire de Vasco ou le scandaleux masochisme!» ${ }^{19}$ :

Nous ne croyons pas du tout au succès du spectacle antimilitariste [...] Ce succès, si le mauvais goût et le défaitisme d'un certain public l'assuraient malgré tout, nous le déplorerions. [...] D'abord parce que Histoire de Vasco de l'écrivain libanais Georges Schehadé appartient à un genre qui nous a toujours paru détestable, avec sa poésie en contre-plaqué,

16 Ebda. S. 121.

17 Ebda. S. 171.

18 Jacques Lemarchand: Histoire de Vasco. In: La Nouvelle Nouvelle Revue Française 48 (1.12.1956), S. 1069-1073. Zit. nach: Georges Schehadé: Le Théâtre du Poète. Correspondances dramatiques et dossier de réception. Herausgegeben von David Martens. Paris: Honoré Champion 2012, S. 375-378.

19 Leon Treich: Histoire de Vasco ou le scandaleux masochisme! In: L'Aurore (08.10.1957), S. 4. Zit. nach: Georges Schehadé: Le Théâtre du Poète, S. 391. Vgl. Treichs nationalistische Studie: L'Esprit français. Paris: Éditions de France 1943. 
sa lourde fantaisie et son anticonformisme pour snobinettes. Ensuite parce que nous ne parvenons pas, [...] à oublier que nos soldats se battent encore en Algérie, et y meurent.

Sartre hatte Texten, die keine konkrete Stellung zu den politischen Konflikten ihrer Zeit nehmen, ihr widerständiges Potential abgesprochen. ${ }^{20}$ Das Euvre von Georges Schehadé, das die Zeitgeschichte in einer universalisierenden Ästhetik aufhebt, widerspricht Sartres Vorstellung. Die Texte des Autors verwandeln die Erfahrung von Migration in eine spezifisch ästhetische Erfahrung, die sich der Logik des Nationalen, die das von Kolonialkriegen geprägte Frankreich dominiert, in dezidierter Weise entzieht, ohne dabei unpolitisch zu sein.

Wie instabil die Begriffe von nationaler Herkunft aber auch von geographischer Orientierung bei Georges Schehadé sind, wird deutlich in dem Theaterstück L'Émigré de Brisbane von 1965. ${ }^{21}$ Darin geht es um die Rückkehr zweier Emigranten in das fiktive Dorf Belvento, Sizilien: Der erste sieht den Dorfplatz, und stirbt, ohne ein Wort gesagt zu haben. Bei der Leiche finden sich ein Geldbeutel sowie ein Schild mit dem Hinweis, dass er seinen unehelichen Sohn habe treffen wollen, der inzwischen 20 Jahre alt sein müsse. Drei Mütter des Dorfes kommen für diese Affaire infrage: Rosa Picaluga kann ihren Mann schnell besänftigen, er sieht auf einmal, wie schön sie noch ist, versöhnt gehen sie wieder ab. Laura Scaramella wird von ihrem eifersüchtigen Mann fast in den Wahnsinn getrieben, kann ihn aber ebenfalls wieder beruhigen. Der Mann von Maria Barbi hält seine Frau für komplett unschuldig, will sie aber überzeugen, eine Affaire mit dem Toten vorzutäuschen und auf diese Weise das Erbe zu erschleichen. Als Maria sich weigert, ihre Ehre für Geld zu verraten, wird sie von ihrem Mann ermordet. Picaluga hat den Plan aber belauscht und macht sich seinerseits auf, um den Mörder und Lügner Barbi zu erschießen und die Ordnung wiederherzustellen.

Erst die Rahmenhandlung bietet Aufklärung: Im letzten Akt bringt der Kutscher einen zweiten Emigranten nach Belvento. Im Dialog der beiden erfährt der Zuschauer, dass es sich gar nicht um das gewünschte Ziel handelt, und der Kutscher gibt zu, den Fahrgast zu einem näheren, schöneren Ort gebracht zu haben: «Aus ästhetischen Gründen», und weil das Pferd Coco schon so alt sei. Das Motiv erinnert an zwei Verse aus einem Gedicht von Schehadé: «Si jamais tu reviens en terre natale / À pas lents comme un cheval dont le soir accroît la fatigue». ${ }^{22}$ So wird auf einmal deutlich, dass auch der erste Besucher niemals Teil

20 Vgl. das Kapitel: Qu'est-ce qu'écrire? In: Jean-Paul Sartre: Qu'est-ce que la littérature? Paris: Gallimard 1948.

21 Georges Schehadé: L'Émigré de Brisbane. Pièce en neuf tableux. Paris: Gallimard 1965.

22 Georges Schehadé: Poésies V, IX, 1985. Die Gedichtbände Schehadés erschienen alle in Paris unter dem Titel Poésies I - VI, in den Jahren 1938, 1948, 1949, 1951, 1972 und 1985. Nur der Band 
der Dorfgemeinschaft von Belvento gewesen ist und die Affaire deshalb nicht aufgeklärt werden konnte. Die Erfahrung des Fremdseins am vermeintlichen Herkunftsort hatte den tödlichen Schock verursacht. Der Kutscher war zum Zeitpunkt des Todes aber schon wieder abgefahren und ahnt nicht, welch blutiges Drama sich inzwischen abgespielt hat. Der zweite Besucher hingegen verzeiht dem Kutscher und kann den Ausflug als ästhetische Erfahrung genießen.

Während das surrealistische Theaterstück Histoire de Vasco stärker avantgardistische Merkmale aufweist, fokussiert der Autor in L'Émigré de Brisbane die Thematik der Auswanderung und einer unmöglichen Rückkehr. Anhand der Zusammenführung von Migration und Avantgarde im Werk Schehadés konnte sich diese künstlerische Bewegung ihrer konkreten politischen Bedeutung wieder bewusstwerden.

\section{Freiheit und Gleichheit auf Arabisch: Zum Werk von Leila Baalbaki (1936*)}

Auch dem Werk von Leila Baalbaki ist bislang die Anerkennung als Ausdruck geistigen Widerstands verwehrt geblieben. Dies zeigt sich unter anderem an dem Umstand, dass sie in Frankreich nur im Vergleich mit französischen Schriftstellerinnen Erwähnung findet: So heißt es oft, sie sei die Colette oder die Françoise Sagan des Libanon, und beeinflusst von den Schriften Simone de Beauvoirs. ${ }^{23}$ Die Werke der französischen Avantgarde können dabei als Folie dienen, um das

Poésies VII wurde 1998 posthum in Beirut veröffentlicht. Vgl. für einen Überblick über das Werk: Jacqueline Michel: Le Pays sans nom. Dhôtel, Supervielle, Schehadé. Paris: Lettres modernes 1989. Kap. 3: «Georges Schehadé et la capitale fabuleuse», S. 103-139. Heribert Becker ist der Meinung, die Welt habe keinen Eingang in die Verse Schehadés gefunden (Ders.: Surrealismus levantinisch). Dem widersprechen die Gedichte selbst. Ein Beispiel wäre die Erwähnung eines Generals in Spanien während des Spanischen Bürgerkriegs: «Tu lis qu'en Espagne un général lève des armées / Et tu songes à des fanfares éparpillées». In: Georges Schehadé: Les Poésies. Paris: Gallimard 2009. <http://revuedepoesie.blog.lemonde.fr/category/domaine-libanais/schehade-georges/> [10.08.2017].

23 Vgl. Michel Barbot: Etoile du Liban. In: Simoun 32 (1961), S. 38-46, hier S. 89, sowie: Katja Ghosn: Leila Baalbaki, l'émancipation faite femme. In: L'orient littéraire (01.04.2010) <http:// www.lorientlitteraire.com/popup.php?n_id=4960\&cid=7> [12.08.2017]. Leila Baalbaki zitiert Sagan in ihrem Essay: Nous sans masques ou la jeunesse arabe dévoilée. Übersetzung von Michel Barbot. In: Revue Orient, troisième trim. (1959), S. 145-163, hier S. 155. Sfeir zitiert ein Interview mit Baalbaki, in dem die Autorin den Unterschied feministischer Ansprüche in Frankreich und im Libanon kommentiert: während Frauen in Frankreich dafür kämpften, unehelich mit einem Mann zusammenleben zu dürfen, wären die Frauen im Libanon schon glücklich, wenn man sie allein 
kritische Potential des Schreibens von Leila Baalbaki herauszuarbeiten. Die Autorin hatte an der Jesuitischen Université Saint-Joseph de Beyrouth studiert und war wie alle libanesischen Intellektuellen bilingual. ${ }^{24}$ Meine These $\mathrm{zu}$ ihrem Euvre lautet, dass Baalbaki in ihren Büchern die republikanischen Werte Freiheit und Gleichheit in eine avantgardistische arabische Literatursprache zu transformieren und feministisch aufzuladen suchte.

Die Autorin war 1936 in eine traditionell gläubige schiitische Familie geboren worden und in Beirut aufgewachsen. Schon im Alter von 14 Jahren begann sie, erste Texte unter einem Pseudonym zu publizieren. Eigenen Aussagen zufolge musste sie drei Monate in einen Hungerstreik gehen, bis der Vater ihr erlaubte, die Schule weiter zu besuchen. ${ }^{25}$ Nach einem Studium der arabischen Literatur arbeitete Baalbaki ab 1957 als Sekretärin am Libanesischen Parlament. ${ }^{26} 1958$ veröffentlichte sie im Alter von 22 Jahren ihren ersten Roman Ana Ahya (Dt. Ich lebe). Daraufhin wurde sie für ein Stipendium nach Paris eingeladen, wo ihr Buch 1961 in einer französischen Übersetzung erschien. ${ }^{27}$ Ana Ahya ist ganz aus der Perspektive der neunzehnjährigen Protagonistin Lina Fayyad geschrieben und kann als das Dokument einer Suche nach Freiheit in zahlreichen inneren Monologen gelesen werden. Schon die Form und die Fokalisierung des Textes galten traditionalistischen Lesern als Provokation: Aufgrund der offenen Darstellung der

shoppen gehen lassen würde. Vgl. George Sfeir: The Contemporary Arabic Novel. In: Daedalus 95, 4 (1966), S. 941-960. Darin: Kap. V. «The female revolt», S. 957-960.

24 Für eine Studie zur Sprachwahl vor dem Hintergrund der gemeinsamen Geschichte von Frankreich und dem Libanon vgl. Michelle Hartman: Subversions from the borderlands. Readings of intertextual strategies in contemporary Lebanese women's literature in Arabic and French. Oxford: University of Oxford Press 1998, bzw. Dies.: Native Tongue, Stranger Talk: The Arabic and French Literary Landscapes of Lebanon. New York: Syracuse University Press 2014.

25 Vgl. Joseph T. Zeidan: Arab Women Novelists. The Formative Years and Beyond. Albany: SUNY Press 1995. Darin Kap. XVIII: «Layla Ba’labakki’s Rebellion: Two Novels», S. 96-104, hier S. 96.

26 Vgl. «Layla Baalbakki», arabwomenwriters.com [06.08.2017], sowie Katja Ghosn: Leila Baalbaki.

27 Vgl. ebda., sowie Leila Baalbaki: Je vis! Paris: Éditions du Seuil (1958) ${ }^{21961 . ~ E r s t e ~ U ̈ b e r-~}$ setzung von V. Monteil (vgl. das Vorwort von Barbot in: Nous sans masques. 1959). Leila Baalbaki: Ich lebe. Übersetzung von Leila Chamaa. Basel: Lenos Verlag 1994. Iman Humaydan, Leiterin des PEN Libanon, wunderte sich 2017 darüber, dass die Romane Leila Baalbakis trotz ihrer Prominenz in der arabischen Welt noch nicht ins Englische übersetzt wurden. Vgl. Marcia Lynx Qualey: Renaissance in Four Voices. Four Women Writers Celebrated in Beirut. In: Arabic Literature and Translation (06.07.2016). <https://arablit.org/2016/07/06/renaissance-in-four-voices-four-women-writers-celebrated-in-beirut/> [18.08.2017]. Die Arab Writers Union setzte den Roman Ana ahya immerhin auf Platz 17 der Top 105 arabischen Romane des 20. Jahrhunderts. Vgl.: <https:// arablit.org/for-readers/top-105/> [18.08.2017]. 
Hoffnungen und Träume einer jungen Frau erntete das Buch scharfe Kritik. ${ }^{28}$ Im Libanon wurde es zensiert; im Irak wurde es aufgrund der politischen Aussagen vollkommen verboten. $^{29}$

Offiziell war Baalbaki in den Jahren 1959 und 1960 an der Sorbonne eingeschrieben, allerdings verbrachte sie wohl die meiste Zeit in den Pariser Cafés, «en plein bouillonnement de l'existentialisme». ${ }^{30}$ Ihre gedankliche Unabhängigkeit zeigt sich auch in dem Essay Nahnu bila aqni'a (Dt. Wir ohne Masken) von 1959, in dem sich Leila Baalbaki für eine straffreie Auseinandersetzung der jungen arabischen Generation mit ihren sexuellen Identitäten starkmacht. Das Freiheitsideal wird dabei an den Ländern gemessen, in denen die Werte Freiheit und Gleichheit offiziell bestimmend sind. Die französische Übersetzung trägt den Titel: Nous sans masques ou la jeunesse arabe dévoilée. ${ }^{31}$

Au pays de Françoise Sagan, au pays d'Elvis Presley, les amoureux flânent sur les trottoirs paisiblement, ils prennent place dans les cafés en toute tranquillité, ils peuvent se chuchoter des mots tendres, s'adresser des reproches, se réconcilier à bouche que veux-tu: personne ne roule des yeux éberlués, personne ne crache son dégoût, personne ne les montre du doigt ou ne les menace. ${ }^{32}$

Die Situation der arabischen Jugend sei dabei im Vergleich zu den Möglichkeiten der europäischen und der amerikanischen Jugend dramatisch unfrei: «Nous sommes plus durs, plus violents, plus infortunés que les jeunes d'Europe et d'Amérique, parce que nous livrons un combat sans merci pour la liberté - sur les plans de l'individu, de l'État et du peuple». ${ }^{33}$ Im weiteren Verlauf der Rede fordert die Autorin neben einer freiheitlicheren Erziehung, die sie als ersten Schritt zur Auflockerung des Konservativismus versteht, auch politische Maßnahmen: unter anderem verlangt sie die Emanzipation der „kleinen“ Staaten aus dem kolonialistischen Machtverhältnis, sowie die Heraushaltung der „großen“ Staaten aus fremden Angelegenheiten. ${ }^{34}$ Baalbaki unterscheidet dabei zwischen Erbe und Geschichte. Traditionen würden in Stein gemeißelt, während die historische Vergangenheit verleugnet werde: «ainsi pleure notre passé». Die koloniale Erfahrung beschreibt die Autorin als «grausames Gespenst»: «Ces pays bafouaient tous ceux

28 Vgl. zur Form des Romans als Novum in der arabischen Literaturgeschichte: George Sfeir: The Contemporary Arabic Novel.

29 Michel Barbot: Etoile du Liban.

30 Katja Ghosn: Leila Baalbaki, S. 3.

31 Leila Baalbaki: Nous sans masques, S. 145-163.

32 Ebda. S. 155.

33 Ebda. S. 155.

34 Ebda. S. 158. 
qui se faisaient l'écho de notre sentiment en diffusant les éléments de civilisation, en éclairant les esprits; enseigner au monde l'horreur de leur spectre était alors la première leçon du cours de liberté.» ${ }^{35}$ Es gelte, die Grausamkeiten kolonialer Geschichte anzuerkennen, und zugleich die französische Geschichte beim Wort zu nehmen, um die Einlösung von Liberté, Égalité und Fraternité zu fordern.

In ihrem zweiten, religionskritischen Roman Al-Aliha al-mamsucha (Dt. Die deformierten Götter) von 1960 geht es ebenfalls um die Freiheit der Frau. ${ }^{36}$ Von den Kurzgeschichten aus Safinatu hanan ila al-qamar (Dt. Ein Raumschiff voller Zärtlichkeit zum Mond) von 1963 heißt es, sie seien inspiriert von dem Aufenthalt in Frankreich. ${ }^{37}$ Die Ausgaben dieses Bandes wurden im Libanon in jedem Laden einzeln konfisziert und die Autorin kam noch im gleichen Jahr wegen «Gefährdung der öffentlichen Moral» vor Gericht. Obwohl sie die Prozesse schließlich gewann, publizierte Baalbaki danach keine weiteren literarischen Texte. ${ }^{38}$ Über ihr Leben weiß man kaum etwas, außer dass sie zu Beginn des Bürgerkriegs den Libanon verließ und in London lebte. Auf Kontaktanfragen reagiert die Autorin seit Jahren nicht mehr. ${ }^{39}$ Die Hoffnung auf eine mögliche posthume Veröffentlichung weiterer Texte bleibt äußerst vage. ${ }^{40}$ Laut der Literaturwissenschaftlerin Roseanne Khalaf handelt es sich bei Baalbaki um die erste Autorin, die ihre Geschichten in der ersten Person Singular erzählte - für die arabische Prosa war dies eine unschätzbare Innovation. ${ }^{41}$ Khalaf betont die Seltenheit der weiblichen Perspektive, zumal Leila Baalbaki einen sehr offenen Umgang mit dem Thema Sexualität gewagt hatte. Dies sei umso bewundernswerter, weil sie aus einer sehr

35 Ebda. S. 160.

36 Joseph Zeidan bezeichnete das Buch als poetischer und kohärenter als die assoziative Erzählung Ana Ahya, vgl. Joseph T. Zeidan: Arab Women Novelists, S. 102. Michel Barbot vergleicht den Roman mit dem Tagebuch von Katherine Mansfield und beschreibt Baalbakis Stil als noch unnachgiebiger: «avec plus d'intransigeance et de savagerie» (Michel Barbot: Etoile du Liban, S. 42).

37 Vgl. Leila Baalbaki: A Spaceship of Tenderness to the Moon. Übersetzung von Denys JohnsonDavis. In: Roseanne Saad Khalaf (Hg.): Hikayat: Short Stories by Lebanese Women. London u. a.: Telegram 2006.

38 Vgl. Joseph T. Zeidan: Arab Women Novelists, S. 103.

39 Vgl. NOW Lebanon: Talking To: Roseanne Khalaf (25.04.2008). <https://now.mmedia.me/lb/ en/interviews/talking_to_roseanne_khalaf $>$ [06.08.2017].

40 Ebda. Khalaf (25.04.2008): «And then, after she had told her stories, she didn't stay on the scene to sort of (fight for women's rights) or do anything like that. She just got her voice out there and disappeared.»

41 Es handelt sich um die erste Ich-Erzählung einer Frau in der arabischen Literatur. Vgl. auch Joseph T. Zeidan: Arab Women Novelists, S. 99. 
konservativen schiitischen Familie kam. ${ }^{42}$ Im Unterschied zu früheren arabischen Schriftstellerinnen habe Leila Baalbaki nicht versucht, männliches Schreiben zu imitieren, sondern eine eigene Stimme gefunden. ${ }^{43}$

\section{Ana ahya (1958) - Widerstand des Individuums}

Der Roman Ana ahya (Dt. Ich lebe) gilt als das wichtigste Werk Leila Baalbakis und soll daher im Folgenden mit Blick auf das Verhältnis zu Frankreich diskutiert werden. Das Buch beschreibt den Kampf der Protagonistin Lina gegen Sexismus im Arbeitsalltag, gegen die patriarchale Vereinnahmung durch den Vater und gegen die traditionellen Werte der Mutter. Der erste Befreiungsversuch der Romanheldin ist ein Kurzhaarschnitt: Lina trennt sich mit den Locken zugleich von einem Verständnis von Weiblichkeit, in dem sie nur als «Heiratsware» gesehen würde. Ihr zweiter Schritt in die Unabhängigkeit ist die heimliche Arbeit als Sekretärin - aber der Versuch scheitert: Die junge Frau wird nicht ernst genommen, und der Arbeitgeber verrät sie an ihren Vater. ${ }^{44}$ Auch die Rendezvous mit Baha, einem irakischen Kommilitonen und überzeugten Kommunisten, laufen ins Leere: echte Zärtlichkeit kommt nie zustande und die erträumte Zukunft als Rebellin an seiner Seite entpuppt sich als Projektion. ${ }^{45}$ In ihrem Elternhaus stört sich Lina sowohl an der Unterwürfigkeit der Mutter und deren Selbstaufgabe in

42 Vgl. Khalaf (25.04.2008): «Leila Baalbaki was... almost imprisoned, because her topics focused on sex and sexuality - and from a woman's point of view. She was accused of corrupting the young, and they tried to imprison her. There was a court case against her. She won in the end, but she was from the South and a very conservative family. So for her, it was quite amazing to be able to do what she did.»

43 Die Literaturwissenschaftlerin verortet Leila Baalbaki in einer Reihe mit Emily Nasrallah und Rima Alameddin. Letztere wurde am 01.01.1963 im Alter von 22 Jahren an ihrem Geburtstag erschossen. Vgl. NOW Lebanon: Talking To: Roseanne Khalaf (25.04.2008). Vgl. zu Emily Nasrallah (Drusin, 1931^): <http://www.emilynasrallah.com/english/biography.html> [08.08.2017]. Rima Alamuddin (1941-1963, ebenfalls Drusin) lebte zeitweise in England und schrieb auf Englisch. Vgl.: $<$ https://www.goodreads.com/author/show/3463539.Rima_Alamuddin> [08.08.2017].

44 Accad vermutet eine Presseagentur, dies wird aber an keiner Stelle spezifiziert. Vgl. Evelyne Accad: Arab women's literary inscriptions: a note and extended bibliography. In: College Literature 22, 1 (Februar 1995), S. 172-180. Eine überarbeitete Version des Textes erschien 2010 in der Canadian Review of Comparative Literature, S. 89-109.

45 Evelyne Accad: Arab women's literary inscriptions, S. 174: «Although Baha is politically radical, he is socially conservative, does not approve of Lina's freedom of action, and eventually tires of her.» 
der patriarchalen Gesellschaft, als auch an der korrupten Moral des Vaters, der geschäftlich vom Zweiten Weltkrieg profitiert: ${ }^{46}$

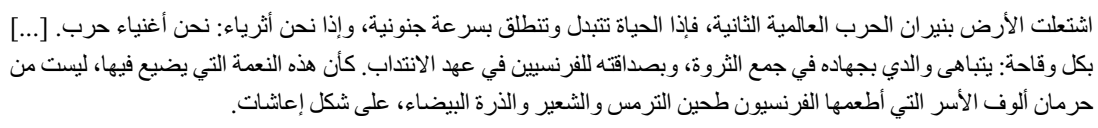

Das Feuer des Zweiten Weltkriegs entbrannte auf der Erde, und siehe da, das Leben wandelte sich und verlief in rasendem Tempo. Und nun sind wir reich! Wir sind Kriegsgewinnler! [...] In aller Unverfrorenheit prahlt mein Vater mit seiner Fähigkeit, Reichtümer anzuhäufen, und mit seiner Freundschaft zu den Franzosen während der Kolonialzeit. Als beruhe der Wohlstand, in dem er schwimmt, nicht auf der Not Tausender von Familien, die von den Franzosen nur Mehl aus Lupinen, Gerste und [...] Hirse zu essen bekommen hatten. ${ }^{47}$

In der direkten Anklage des Vaters versteckt sich eine Kritik am Verhalten der französischen Besatzungsmacht während des Zweiten Weltkriegs. Im Vordergrund steht jedoch die Positionierung der Libanesen selbst.

Der Roman kommentiert sowohl den Konflikt um den Suezkanal 1956 sowie die Libanonkrise von 1958. Die Verstaatlichung des Suezkanals ${ }^{48}$ war Anlass für den Angriff von Frankreich, Großbritannien und Israel auf Ägypten. Die ehemaligen Kolonialmächte sahen ihren Einfluss schwinden und gewannen mit Israel einen strategischen Partner vor Ort. In einer während des Kalten Krieges einmaligen Koalition erwirkten jedoch ausgerechnet die USA und die UdSSR den Abzug der Truppen und verhinderten den Sturz des ägyptischen Präsidenten Gamal Abdel Nasser. Während der Monate der Libanonkrise 1958 spaltete sich das Land in Befürworter einer prowestlichen Politik auf der einen Seite, und proarabische Stimmen auf der anderen. Die Protagonistin Lina reflektiert diesen politischen Konflikt auf persönlicher Ebene und verortet sich selbst dialektisch: ohne dies als Widerspruch zu sehen, vertritt sie einerseits ein Selbstverständnis als Libanesin, und kämpft zugleich im ganzen Roman für die Freiheit und Gleichberechtigung, die ihr eine weltoffene, republikanische Gesellschaft bieten würde.

Auch ganz alltägliche Dinge werden zum Politikum. So fragt Lina sich zum Beispiel, warum die Mutter versucht, sie mit französischen Gerichten zu bestechen, statt gefüllte Zucchini, Tabbuleh oder Kibbe zu kochen. Im arabischen

46 Leila Baalbaki: Ana Ahya. London/Beirut: Al Jaber Foundation, Unesco 2010 (Kitab fi Jarida), S. 10. < http://www.kitabfijarida.com/pdf/145.pdf> [05.08.19].

47 Leila Baalbaki: Ich lebe, S. 31.

48 Vgl. ebda., S. 39. 
Original betont sie immer wieder, dass sie keine Französin sei (in der deutschen Übersetzung wurde die «Französin» durch eine «Europäerin» ersetzt): ${ }^{49}$

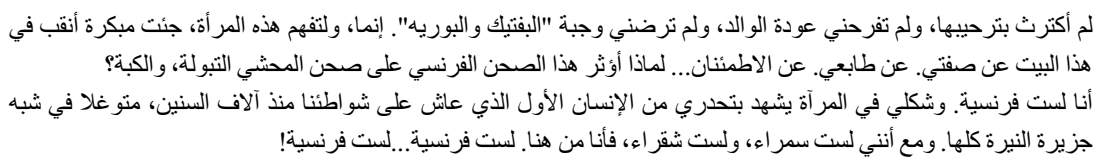

Ich scherte mich nicht um ihre freundliche Begrüßung, über Vaters Rückkehr freute ich mich auch nicht, und das «Beefsteak mit Püree» machte mich erst recht nicht glücklich. Wann begreift diese Frau endlich, dass ich heimkomme, um hier im Haus nach mir zu suchen? Nach meiner Natur. Nach Sicherheit. Außerdem, wieso soll ich eigentlich dieses europäische [französische] Gericht lieber mögen als Machschi, Tabbula und Kubba? Ich bin keine Europäerin [Französin]. Wenn ich mich im Spiegel betrachte, weiß ich, dass ich vom ersten Menschen abstamme, der vor tausenden von Jahren an unseren Küsten lebte und die ganze wunderbare Halbinsel besiedelte. Und obwohl ich weder dunkelhaarig noch blond bin, bin ich von hier. Ich bin keine Europäerin [Französin.$^{50}$

Der bewusste Blick in den Spiegel ist in diesem Fall als Selbstermächtigung zu deuten: Lina wehrt sich gegen Zuschreibungen von außen, sie möchte sich selbst gehören. In der folgenden Szene beschreibt die Protagonistin die Kombination von amerikanisch eingerichteten Zimmern und dem traditionellen arabischen Wohnzimmer mit Gebetsteppichen, Samtkissen, einem glühenden Kupferofen und einer Nargileh. Zwischen diesen zwei Welten fühlt sie sich verloren: ${ }^{51}$

$$
\text { أنا في بيتنا ضائعة: لست شرقية، ولست غربية. لست حرة، ولست مستعبدة، لست شقر اء، ولست سمر اء! }
$$

In unserem Haus fühle ich mich verloren: ich bin keine Orientalin und auch keine Europäerin [bzw. Ich bin weder aus dem Osten noch aus dem Westen, Anm. cvw]. Ich bin weder frei, noch bin ich unterdrückt [versklavt]. Ich bin weder blond noch braun. ${ }^{52}$

Linas Mitarbeiter in der Agentur hat den Konflikt für sich gelöst. Statt nationale Unternehmen mit seiner Arbeitskraft zu unterstützen, bereichert er sich am Geld der ehemaligen Kolonialisten und rechtfertigt sich dafür wie folgt: ${ }^{53}$

49 Leila Baalbaki: Ana Ahya, S. 19.

50 Leila Baalbaki: Ich lebe, S. 68.

51 Leila Baalbaki: Ana ahya, S. 19.

52 Leila Baalbaki: Ich lebe, S. 68.

53 Leila Baalbaki: Ana ahya, S. 18. 


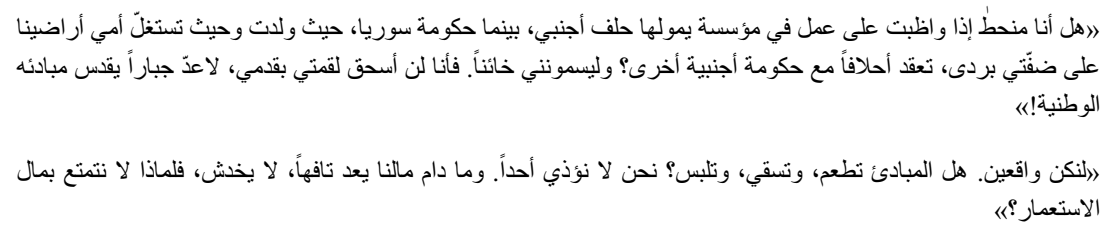

«Bin ich etwa verkommen», wollte er wissen, «wenn ich für eine Firma arbeite, die von einem ausländischen Bündnis finanziert wird, während in Syrien, wo ich geboren wurde und wo meine Mutter aus unseren Ländereien am Barada profitiert, die Regierung mit einem anderen ausländischen Partner paktiert? Soll man mich doch einen Verräter nennen. Ich denke nicht daran, mein täglich Brot mit Füßen zu treten, um als Held zu gelten, der seine nationalen Prinzipien heiligt! [...] Seien wir ehrlich. Verschaffen einem die Prinzipien etwa Essen, Trinken und Kleidung? Wir tun keinem weh. Und solange der einzelne bei uns als ein Nichts gilt [bzw. solange unser Geld nichts wert ist], können wir uns doch ruhig am imperialistischen [kolonialistischen] Vermögen bereichern!» ${ }^{54}$

Der hier zum Ausdruck gebrachte Konflikt zwischen den Unabhängigkeitsbestrebungen der arabischen Länder und einer republikanischen Welt, in der eine junge Frau problemlos alleine ins Kino gehen, rauchen, politische Meinungen und Liebesbeziehungen haben könnte, verkörpert sich in der Romanheldin. Lina wehrt sich gegen die schlichte Imitation französischer Alltagskultur und sehnt sich zugleich nach der Freiheit westlicher Demokratien. Diese dialektische Bezugnahme auf Frankreich zieht sich durch Baalbakis gesamtes Werk.

\section{VI Ästhetik und Rebellion}

Leila Baalbaki kämpfte in ihren Texten für eine Republikanisierung ihrer Lebenswirklichkeit, ohne dass diese als Zugeständnis an die Einflüsse der ehemaligen Kolonialmacht und damit als Verrat am kulturellen Erbe des Libanon gesehen würde. Während die Biographie Schehadés den Magnetismus der Stadt Paris verdeutlicht, zeigt sich im Werk von Baalbaki die Zentrifugalkraft avantgardistischer Bewegungen. Dabei geht es weniger um eine Unterscheidung zwischen Zentrum und Peripherie, sondern die durch Paris symbolisierten Werte - Freiheit, Gleichheit, Rebellion und Bohème - werden als Teil der eigenen Geschichte verstanden. Anstelle einer Migration der Autorin steht in diesem Kontext die Migration des avantgardistischen Impulses im Vordergrund. ${ }^{55}$ Eine erneute Kolo-

54 Leila Baalbaki: Ich lebe, S. 67.

55 Vgl. Mieke Bal: Travelling Concepts in the Humanities. A Rough Guide. Toronto: University of Toronto Press ('2002) 2012. 
nialisierung vermeidet Baalbaki, indem sie die französische Literatur nicht $\mathrm{zu}$ imitieren sucht, sondern der avantgardistischen Bewegung mit ihrem Werk gewissermaßen einen arabischen Pass ausstellt. Dadurch wird auch die politische Geste verständlich: die Autorin widersetzt sich sowohl der französischen Ästhetik als auch den gegebenen Möglichkeiten der arabischen Literatur. Gerade diese Unangepasstheit ist es, die ihrem Werk die spezifische avantgardistische Qualität verleiht.

Mit rebellischen Protagonistinnen wie Lina Fayyad und poetischen Antihelden wie Vasco widerstehen Leila Baalbaki und Georges Schehadé den eindimensionalen politischen Narrativen ihrer Zeit. Schehadé experimentierte dabei vor allem in seinem absurden Theater mit den ästhetischen Formen der Avantgarde, während Baalbaki insbesondere die avantgardistische Logik des Aufbruchs herkömmlicher Traditionen auf einer inhaltlichen Ebene für ihr Schreiben wirksam machte. Ihre assoziativen inneren Monologe und ihre teilweise überraschend harte Sprache gelten als stilistische Revolution in der arabischen Prosa; die unerhörte Perspektive der weiblichen Ich-Erzählerin provozierte die libanesische Öffentlichkeit bis hin zu juristischen Maßnahmen gegen die Autorin. In diesem Zusammenhang kann der avantgardistische Impuls durchaus als politische Aufforderung verstanden werden. Indem andere Ästhetiken ausgelotet werden, geht es darum, Geschichte infrage zu stellen und das Eigene neu zu verorten.

\section{Literaturverzeichnis}

\section{Primärliteratur}

Baalbaki, Leila: Ana ahya! Beirut: Dar Majallat Chir 1958.

Baalbaki, Leila: Je vis! Übersetzung von Michel Barbot. Paris: Éditions du Seuil 21961.

Baalbaki, Leila: Ich lebe. Übersetzung von Leila Chamaa. Basel: Lenos Verlag 1994.

Baalbaki, Leila: Ana Ahya. London/Beirut: Al Jaber Foundation, Unesco 2010 (Kitab fi Jarida). <http://www.kitabfijarida.com/pdf/145.pdf> [05.08.19]

Baalbaki, Leila: Nous sans masques ou la jeunesse arabe dévoilée. Übersetzung von Michel Barbot. In: Revue Orient, troisième trim. (1959), S. 145-163.

Baalbaki, Leila: Mes ombres et la nuit. Übersetzung von Michel Barbot. In: Simoun. Revue littéraire bimestrielle 32 (1961), S. 47-49.

Baalbaki, Leila: A Spaceship of Tenderness to the Moon. Übersetzung von Denys Johnson-Davis. In: Roseanne Saad Khalaf (Hg.): Hikayat: Short Stories by Lebanese Women. London u. a.:

Telegram 2006.

Schehadé, Georges: Histoire de Vasco. Pièce en six tableaux. Paris: Gallimard, 1957.

Schehadé, Georges: L'Émigré de Brisbane. Pièce en neuftableux. Paris: Gallimard, 1965.

Schehadé, Georges: Les Poésies. Paris: Gallimard, 1969. <http://revuedepoesie.blog.lemonde. $\mathrm{fr} /$ category/domaine-libanais/schehade-georges/> [10.08.2017]. 
Schehadé, Georges: Poesie I-VII, französisch - deutsch. Übersetzung von Jürgen Brôcan. Berlin: Hans Schiler 2006.

\section{Sekundärliteratur}

Accad, Evelyne: Arab women's literary inscriptions: a note and extended bibliography. In: College Literature 22, 1 (Februar 1995), S. 172-180.

Bal, Mieke: Travelling Concepts in the Humanities. A Rough Guide, Toronto u. a. (12002) ${ }^{2} 2012$. Barbot, Michel: Etoile du Liban. In: Simoun 32 (1961), S. 38-46.

Becker, Heribert: Surrealismus levantinisch. Lyrik zwischen Symbolismus und Surrealismus der libanesische Dichter Georges Schehadé. Qantara.de 2006. .<http://de.qantara.de/ inhalt/georges-schehade-surrealismus-levantinisch> [08.08.2017].

Carmody, Francis J.: Les Poésies by Georges Schehadé. In: The French Review 26, 2 (Dezember 1952), S. 145-147. <http://www.jstor.org/stable/382870> [03.08.2017].

Delas, Daniel: La mémoire et l'éphémère dans la poésie de Georges Schehadé. In: Neohelicon XXXIII, 1 (2006), S. 131-137.

Ghosn, Katja: Leila Baalbaki, l'émancipation faite femme. In: L'orient littéraire (01.04.2010). <http://www.lorientlitteraire.com/popup.php?n_id=4960\&cid=7> [12.08.2017].

Hartman, Michelle: Subversions from the borderlands: Readings of intertextual strategies in contemporary Lebanese women's literature in Arabic and French. Oxford: University of Oxford Press 1998.

Hartman, Michelle: Native Tongue, Stranger Talk: The Arabic and French Literary Landscapes of Lebanon. New York: Syracuse University Press 2014.

Kerdraon, Joel: Georges Schéhadé (1905-1989). Eintrag auf: A la lettre. Mit Werkübersicht. <http://www.alalettre.com/schehade.php> [08.08.2017].

Knapp, Bettina: He who dreams diffuses into air.... In: Yale French Studies 29, S. 108-115.

Lynx Qualey, Marcia: Renaissance in Four Voices. Four Women Writers Celebrated in Beirut. In: Arabic Literature and Translation (06.07.2016). <https://arablit.org/2016/07/06/renaissance-in-four-voices-four-women-writers-celebrated-in-beirut/> [18.08.2017].

NOW Lebanon: Talking To: Roseanne Khalaf, 25.04.2008. <https://now.mmedia.me/lb/en/interviews/talking_to_roseanne_khalaf> [06.08.2017].

Sartre, Jean-Paul: Qu'est-ce que la littérature? Paris: Gallimard 1948.

Sfeir, George: The Contemporary Arabic Novel. In: Daedalus 95, 4 (1966), S. 941-960. Darin: Kap. V. «The female revolt», S. 957-960.

Zeidan, Joseph T.: Arab Women Novelists: The Formative Years and Beyond. Albany: SUNY Press 1995. Darin Kap. XVIII: «Layla Ba’labakki’s Rebellion: Two Novels», S. 96-104. 